\title{
Influencia del exceso de peso en el éxito de la adenoamigdalectomía en pacientes con hiperplasia adenoamigdalina sintomática
}

\author{
The influence of overweight in the success of adenotonsillectomy in patients \\ with adenotonsillar symptomatic hyperplasia
}

Gerardo Oberreuter $\mathrm{E}^{1}$, Ignacio Cortés $\mathrm{F}^{2}$, Leopoldo Córdova $\mathrm{P}^{2}$, Daniel Muñoz S1', Loreto Carrasco $\mathbf{M}^{1}$.

\begin{abstract}
RESUMEN
Introducción: En población pediátrica con malnutrición por exceso, existe controversia respecto al rol de la hiperplasia adenoamigdalina en la etiología de los trastornos del sueño y de la efectividad de la adenoamigdalectomía sobre dicha sintomatología.

objetivo: Comparar la efectividad de la adenoamigdalectomía entre pacientes pediátricos eutróficos y con malnutrición por exceso sometidos a adenoamigdalectomía por hiperplasia adenoamigdalina, en relación a la disminución de la sintomatología.

Materiales y método: Estudio retrospectivo mediante revisión de fichas clínicas entre junio de 2016 y enero de 2017 de pacientes operados de adenoamigdalectomía por hiperplasia adenoamigdalina sintomática. Se clasificaron los pacientes de acuerdo a edad y estado nutricional en 4 grupos y se evaluó la resolución de la sintomatología mediante interrogación a padres/tutores.

Resultados: Se incluyeron 98 pacientes, con una edad media de 6,3 años. 44,9\% de Ios pacientes fueron eutróficos y 55,1\% con malnutrición por exceso. El análisis estadístico entre pacientes eutróficos y aquellos con malnutrición no demostró diferencias significativas en relación a la resolución de la sintomatología.

Conclusión: La adenoamigdalectomía por hiperplasia adenoamigdalina sintomática se asocia a una reducción de la frecuencia de roncopatía con pausas en pacientes pediátricos, independientemente del estado nutricional.
\end{abstract}

Palabras claves: Hiperplasia, hipertrofia, adenoamigdalina, adenoamigdalectomía, sobrepeso, obesidad

\begin{abstract}
Introduction: In pediatric population with malnutrition by excess, the role of the adenotonsillar hyperplasia in the etiology of breathing-related sleep disorders, and the effectivity of the adenotonsillectomy for reducing symptoms remains controversial.

Aim: To compare and evaluate the effectiveness of the adenotonsillectomy between eutrophic and malnutrition by excess pediatric patients submitted to adenotonsillectomy by adenotonsillar hyperplasia, regarding to symptoms resolution.
\end{abstract}

1 Servicio Otorrinolaringología. Hospital San Juan de Dios, Santiago, Chile.

2 Interno de Medicina. Facultad de Medicina, Universidad de Chile.

Recibido el 7 de abril, 2018. Aceptado el 3 de junio, 2018. 
Material and method: A retrospective study was performed collecting data from clinical records between June 2016 and January 2017 of patients submitted to adenotonsillectomy by symptomatic adenotonsillar hyperplasia. Patients were assigned to 4 groups according to age and nutrional status and the rate of symptoms resolution was evaluated through interrogation to parents/guardians.

Results: We included 98 patients, with an average age of 6.3 years. A $44.9 \%$ of patients were eutrophic and $55.1 \%$ had malnutrition by excess. Comparing eutrophic and malnutrition by excess patients, no significant differences were observed regarding to symptoms resolution.

Conclusion: The adenotonsillectomy by symptomatic adenotonsillar hyperplasia was associated to an amelioration of the rate of snoring and respiratory pauses during sleeping in pediatric patients, independently of the nutritional status.

Key words: Adenotonsillar, hypertrophy, hyperplasia, adenotonsillectomy, overweight, obesity

\section{INTRODUCCIÓN}

La adenoamigdalectomía (AA) corresponde a una de las cirugías otorrinolaringológicas más frecuentemente realizadas, especialmente en población pediátrica donde se reportan cerca de 530.000 procedimientos anuales en USA ${ }^{1}$. Tradicionalmente, la amigdalitis recurrente correspondía a la indicación más frecuente de AA. En la actualidad, sin embargo, son los trastornos respiratorios del sueño asociados a la hiperplasia adenoamigdalina (HAA) los que constituyen la primera causa de $\mathrm{AA}^{2}$.

Los trastornos respiratorios del sueño incluyen un grupo de desórdenes caracterizados por anormalidades en la respiración y ventilación, manifestados clínicamente desde ronquidos y pausas hasta episodios de apnea y desaturación durante el sueño ${ }^{3}$. En población infantil, la presencia de estos trastornos se ha asociado a consecuencias negativas en el desarrollo cognitivo y comportamiento, tales como peor desempeño académico, dificultad en aprendizaje y disminución en la capacidad de atención ${ }^{4,5}$.

En la etiopatogenia de estos trastornos, se ha demostrado la asociación entre HAA y la presencia de sintomatología durante el sueño ${ }^{3}$. En consecuencia, existe evidencia que ha reportado efectos beneficiosos de la AA sobre las manifestaciones clínicas de estos trastornos, como también posibles efectos positivos en reducir las consecuencias cognitivas asociadas a dichos trastornos y en la calidad de vida de los pacientes intervenidos ${ }^{6-11}$. Sin embargo, la mayoría de estos estudios son no controlados y no poseen un seguimiento a largo plazo.
En población con malnutrición por exceso existe controversia respecto al rol de la HAA en la etiología de la sintomatología asociada a los trastornos respiratorios del sueño. Dicha población ha presentado durante los últimos años un dramático aumento de su prevalencia a nivel global ${ }^{12}$, acarreando consigo un aumento de patologías asociadas. Se ha planteado que en estos pacientes, la presencia de sintomatología respiratoria durante el sueño sería a causa de la compresión producida por el tejido adiposo depositado alrededor del cuello $0^{13-15}$ y una disfunción en el control neuromotor que produciría un mayor colapso de la faring $\mathrm{e}^{16}$. La presencia de HAA en estos pacientes generaría una reducción aún mayor de las vías respiratorias, contribuyendo a la generación de sintomatología respiratoria ${ }^{17}$. Es por ello que el rol de la AA en reducir la sintomatología en pacientes con malnutrición por exceso, es aún materia de estudio y controversia ${ }^{18}$.

Actualmente, pocos estudios han evaluado directamente los resultados posoperatorios de la AA sobre la reducción de la sintomatología en pacientes con malnutrición por exceso e HAA ${ }^{18,19}$.

\section{OBJETIVOS}

El presente trabajo tiene como objetivo evaluar y comparar la efectividad de la AA en pacientes operados por HAA sintomática entre sujetos eutróficos y con malnutrición por exceso en relación a la resolución de la sintomatología asociada. Del mismo modo, y como objetivos secundarios 
se comparó la frecuencia de complicaciones posquirúrgicas y el grado de satisfacción de los cuidadores entre las poblaciones estudiadas.

\section{MATERIAL Y MÉTODO}

Estudio retrospectivo mediante revisión de fichas de pacientes operados de adenoidectomía, amigdalectomía 0 adenoamigdalectomía entre junio de 2016 y enero de 2017 por hiperplasia adenoidea, amigdalina 0 adenoamigdalina sintomática, respectivamente en el Servicio de Otorrinolaringología del Hospital San Juan de Dios. La indicación quirúrgica de amigdalectomía se definió mediante entrevista al cuidador, quien debe evidenciar y describir una roncopatía con pausas durante el sueño del niño de al menos 3 segundos de duración, asociado a un examen físico donde se observen amígdalas de al menos grado 3 según la clasificación de Brodsky. La indicación de adenoidectomía se realizó con la misma entrevista al cuidador, asociado a un estudio endoscópico endonasal donde se observara tejido adenoideo de al menos grado 3 según la clasificación de Parikh, 0 bien mediante palpación que demuestre obstrucción de las coanas por tejido adenoideo durante una amigdalectomía. Estos criterios son utilizados por todo el servicio de otorrinolaringología de nuestro centro. Así, se crearon cuatro grupos en base al estado nutricional medido por IMC/edad en pacientes con mayor 0 igual a 5 años de edad 0 peso/talla en menores de esta edad. En el primer grupo se incluyeron los pacientes eutróficos, en el segundo grupo los pacientes con sobrepeso, en el tercero con obesidad y en el cuarto con obesidad severa, según los criterios diagnósticos de la norma MINSAL 2016. Se evaluó la resolución de la patología mediante interrogación a los padres y/o tutores a través de una encuesta vía telefónica, donde se les preguntó si el paciente dejó de roncar y/o tener pausas respiratorias durante el sueño. Si persistía con ronquidos, se les solicitó detallar si éstos ocurrían diaria u ocasionalmente y definir la intensidad en leve, moderado o severo. Si persistía con pausas, describir el tiempo de duración y frecuencia. Además, se evaluó la presencia de complicaciones posoperatorias, el cambio del estado nutricional de los pacientes y el grado de satisfacción de los padres o tutores a través de esta encuesta.

\section{RESULTADOS}

En el periodo mencionado se recolectaron un total de 157 fichas de pacientes que fueron operados por hiperplasia adenoamigdalina sintomática. De éstos, no fue posible contactar para el seguimiento a 59 porque habían cambiado de número telefónico, dirección, ambos o fue mal ingresado en la ficha médica; lo que nos deja con 98 pacientes para el análisis. El $54 \%$ de los pacientes (53) fueron hombres. El promedio de edad 6,3 años. El diagnóstico nutricional fue de eutrofia en el $44,9 \%$ (45), sobrepeso en el 18,3\% (18), obesidad en el $19,4 \%$ (19) y obesidad severa en el $17,4 \%$ (16).

A nivel global, según lo evaluado mediante interrogación a los cuidadores, el $77,6 \%$ de los pacientes dejó de roncar y tener pausas respiratorias y el $2,4 \%$ ronca diariamente. Todos los pacientes solucionaron las pausas durante el sueño. Para el análisis estadístico se ajustó un modelo de regresión logística ordinal, sobre la mejoría de los síntomas cuyas variables predictoras consideraban el estado nutricional del sujeto. Se utilizó el software estadístico Stata 15.0 (StataCorp 2017. Stata Statistical Software: Release 15. College Station, TX: StataCorp $\mathrm{LLC}$ ), considerando como estadísticamente significativo un valor $p<0,05$. Resultando este análisis sin diferencias significativas entre los distintos grupos.

En cuanto a la variación del estado nutricional de los pacientes, el $61,2 \%$ mantuvo su estado nutricional previo, el 22,4\% lo disminuyó (de sobrepeso u obesidad a eutrofia) y el $16,4 \%$ lo aumentó (de eutrofia a sobrepeso u obesidad). Con respecto a las complicaciones, sólo un paciente presentó sangrado que requirió hospitalización, pero no una reintervención quirúrgica. Esto representa complicaciones en el $1 \%$ de la muestra.

Finalmente, además de responder las preguntas sobre roncopatía con pausas durante el sueño, al pedirle al cuidador que responda qué tan de acuerdo está con la siguiente frase, calificándola del 1 al 10, los resultados fueron los siguientes (se indica el promedio):

La cirugía mejoró el desempeño académico de mi hijo: 7,5. 
La cirugía mejoró la atención de mi hijo: 7,1.

La cirugía mejoró mi calidad de vida: 9,0.

\section{DISCUSIÓN}

En el presente estudio no se observaron diferencias significativas en la frecuencia de resolución de la roncopatía con pausas entre pacientes eutróficos y aquellos con sobrepeso-obesidad, existiendo una respuesta favorable a la intervención independiente del estado nutricional del paciente. Los resultados encontrados en nuestra casuística, pudiesen ser concordantes con estudios en donde se ha descrito en pacientes con exceso de peso y roncopatía con pausas, la reducción posterior a su AA de la sintomatología respiratoria previa, asociada además a mejoría en los parámetros de sueño $0^{19} \mathrm{y}$ saturación ${ }^{20}$.

En la misma línea, sin embargo, se han publicado revisiones en donde se concluye que, si bien existe reducción en la severidad de los trastornos del sueño en pacientes obesos sometidos a AA, este procedimiento raramente es curativo, planteándose como explicaciones el carácter multinivel de la obstrucción generadora de trastornos respiratorios del sueño en los niños obesos (similar a la de los obesos adultos), y por tanto menos susceptible de curar completamente mediante $A A^{18}$.

Las diferencias encontradas entre las publicaciones deben ser analizadas teniendo en consideración una serie de factores clínicos y metodológicos. Inicialmente, destacar la variación que han tenido a lo largo del tiempo las normas de evaluación nutricional de la población pediátrica, las cuales han evolucionado acorde a los cambios demográficos de las sociedades y las condiciones de aplicabilidad en los distintos niveles de atención de los respectivos países. En el caso nacional contamos con una reciente actualización (2016) de los criterios nutricionales, la cual permite una mayor pesquisa de niños con malnutrición por exceso respecto a las normas previas. Lo anterior genera diferencias entre las poblaciones objetivos de investigaciones según los criterios utilizados.

Del mismo modo, la experiencia clínica nos indica la divergencia existente entre los distintos centros hospitalarios respecto a los criterios de intervención quirúrgica de los pacientes obesos con HAA y trastornos del sueño, exigiéndose en algunos medios aumentos de volumen adenoamigdalino superiores a los exigidos en población eutrófica, pudiéndose plantear que el componente obstructivo derivado de la HAA es mayor y por tanto más susceptible de beneficiarse de cirugía. La poca anuencia respecto a los criterios clínicos utilizados para intervenir a este grupo de pacientes, genera poblaciones intervenidas con distintos perfiles de severidad de HAA, y por tanto, con distintos grados de respuesta a la AA.

Pacientes con SAHOS establecido también se beneficiarían de amigdalectomía en términos de reducción del índice apnea-hipopnea ${ }^{21,22}$, siendo el beneficio mayor en pacientes no obesos que obesos ${ }^{23,24}$ y menor en pacientes con comorbilidades neurológicas, adolescentes y anormalidades craneofaciales ${ }^{22}$. En efecto, ha sido descrita una relación inversa entre el grado de obesidad medido por IMC y la reducción de los eventos de apnea nocturnos, perdiéndose el beneficio en aquellos pacientes con obesidad de mayor severidad ${ }^{23,25}$. Por otro lado, otro reporte muestra que la disminución del índice apnea-hipoapnea dependería más del valor inicial (mayor valor, mayor porcentaje de éxito) que del grado de obesidad inicia ${ }^{26}$, siendo esto aún objeto de controversia ${ }^{19}$. De forma interesante, ha sido reportado una reducción en los parámetros inflamatorios (Interleucina-6 (IL-6), IL-18, inhibidor del activador del plasminógeno-1, proteína quimioatrayente de monocitos-1, metalproteinasa de matriz-9 y adropina) asociados a SAHOS posterior a la $A A^{27}$, lo cual aún es materia de estudio y controversia ${ }^{28-30}$, desconociéndose las potenciales implicancias de estos hallazgos en el futuro.

En nuestros resultados no se observó una relación entre presencia de sobrepeso-obesidad y mayor riesgo de complicaciones relacionadas al procedimiento quirúrgico. No obstante, ha sido reportado que pacientes con sobrepeso/obesidad tendrían mayor frecuencia de efectos adversos, relacionados principalmente a la intubación y ventilación perioperatoria ${ }^{31}$, mayor dolor posoperatorio ${ }^{32}$, aumento del riesgo de sangrado ${ }^{33}$, mayor riesgo de eventos respiratorios como neumonía posoperatoria ${ }^{34,35}$, siendo algunos de estos parámetros no explorados en nuestro estudio. Además, la mayor tasa de complicaciones aumenta los costos asociados a la atención ${ }^{36}$. 
Algunos estudios han reportado un aumento del peso en pacientes sometidos a amigdalectomía, aparentemente por una mayor facilidad en la deglución de alimentos ${ }^{37,38}$, siendo aún objeto de controversia $^{39,40}$. Lo anterior no fue observado en nuestros resultados, sin embargo nuestro seguimiento fue de sólo un año, por lo que desconocemos la evolución a futuro de estos pacientes. Lo anterior plantea la necesidad de tener estudios con un mayor periodo de seguimiento respecto a los pacientes intervenidos.

Por último, destacar el alto porcentaje de la población estudiada (55\%) que presenta algún grado de malnutrición por exceso. Lamentablemente, lo anterior no está lejos de los últimos datos epidemiológicos nacionales, en donde se registra en promedio $50,3 \%$ de sobrepeso-obesidad en escolares $^{41}$ y cerca de $74 \%$ en población adulta ${ }^{42}$, de acuerdo a datos del año 2017.

Pese a que en la práctica clínica una meticuIosa historia y examen físico resultan suficientes para el correcto diagnóstico de la HAA y para la determinación de la resolución quirúrgica, es importante destacar que gran parte de la investigación y evidencia disponible en la literatura utilizan la polisomnografía como herramienta de evaluación de los trastornos respiratorios del sueño. La realidad local de nuestro centro hospitalario hace complejo tener acceso a herramientas como la polisomnografía, la cual se constituye como el gold standard en el diagnóstico y seguimiento de

\section{BIBLIOGRAFÍA}

1. Ingram DG, Friedman NR. Toward Adenotonsillectomy in Children. JAMA Pediatr 2015; 169: 1155.

2. Parker NP, Walner DL. Trends in the indications for pediatric tonsillectomy or adenotonsillectomy. Int J Pediatr Otorhinolaryngol 2011; 75: 282-5.

3. Grime C, TAN H. Sleep Disordered Breathing in Children. Indian J Pediatr 2015; 82: 945-55.

4. Smith DL, Gozal D, Hunter SJ, Kheirandish-Gozal L. Frequency of snoring, rather than apneahypopnea index, predicts both cognitive and behavioral problems in young children. Sleep Med 2017; 34: 170-8.

5. Galland B, Spruyt K, Dawes P, McDowall PS, Elder estos pacientes ${ }^{42}$, haciendo dificultosa también la comparación de nuestros resultados. No obstante, y de forma similar a estudios internacionales, el alto porcentaje de satisfacción usuaria ${ }^{43-45}$ y mejora en la percepción de calidad de vida ${ }^{46,47}$ representan aspectos cualitativos positivos que podrían guiar la elección de una alternativa quirúrgica como método de resolución de la sintomatología en ausencia de polisomnografía.

\section{CONCLUSIÓN}

La adenoamigdalectomía por hiperplasia adenoamigdalina sintomática se asocia a una reducción de la frecuencia de roncopatía con pausas a corto plazo en pacientes pediátricos con independencia del estado nutricional. En esta serie de casos se constituye como un procedimiento efectivo, seguro y con un alto grado de satisfacción por parte de los cuidadores respecto a la calidad de vida y desempeño cognitivo de los pacientes intervenidos. Desconocemos los resultados de este procedimiento en el seguimiento a largo plazo de estos pacientes, lo cual debería ser evaluado en futuras investigaciones. Por último, el alarmante incremento de población pediátrica con malnutrición por exceso en los últimos años sustenta la realización de nuevos estudios que evalúen el rol de la $A A$ en reducción de sintomatología asociada a HAA en dicha población.

D, Schaughency E. Sleep Disordered Breathing and Academic Performance: A Meta-analysis. Pediatrics 2015; 136: e934-46.

6. Todd CA, Bareiss AK, McCoul ed, Rodriguez $\mathrm{KH}$. Adenotonsillectomy for Obstructive Sleep Apnea and Quality of Life: Systematic Review and Meta-analysis. Otolaryngol Neck Surg 2017; 157: 767-73.

7. Chervin RD. Sleep-Disordered Breathing, Behavior, and Cognition in Children Before and After Adenotonsillectomy. Pediatrics 2006; 117: e769-78.

8. Garetz SL. Behavior, cognition, and quality of life after adenotonsillectomy for pediatric sleepdisordered breathing: Summary of the literature. Otolaryngol Neck Surg 2008; 138: S19-26. 
9. Tran KD, Nguyen CD, Weedon J, Goldstein NA. Child Behavior and Quality of Life in Pediatric Obstructive Sleep Apnea. Arch Otolaryngol Neck Surg 2005; 131: 52.

10. Goldstein NA, Fatima M, Campbell TF, Rosenfeld RM. Child behavior and quality of life before and after tonsillectomy and adenoidectomy. Arch Otolaryngol Head Neck Surg 2002; 128: 770-5.

11. Mitchell RB, Kelly J. Behavior, neurocognition and quality-of-life in children with sleep-disordered breathing. Int J Pediatr Otorhinolaryngol 2006; 70: 395-406.

12. Wang Y, LobsteIn T. Worldwide trends in childhood overweight and obesity. Int J Pediatr Obes 2006; 1: $11-25$.

13. Horner RL, Mohiaddin RH, Lowell DG, Shea SA, Burman ED, Longmore DB, et al. Sites and sizes of fat deposits around the pharynx in obese patients with obstructive sleep apnoea and weight matched controls. Eur Respir J 1989; 2: 613-22.

14. Shelton Ke, Woodson H, Gay S, Suratt PM. Pharyngeal fat in obstructive sleep apnea. Am Rev Respir Dis 1993; 148: 462-6.

15. Hoffstein V, Zamel N, Phillipson EA. Lung volume dependence of pharyngeal cross-sectional area in patients with obstructive sleep apnea. Am Rev Respir Dis 1984; 130: 175-8.

16. Marcus CL, Katz eS, Lutz J, Black CA, Galster P, CARson KA. Upper airway dynamic responses in children with the obstructive sleep apnea syndrome. Pediatr Res 2005; 57: 99-107.

17. Verhulst SL, Schrauwen N, Haentuens D, Suys B, Rooman RP, Van Gaal L, et al. Sleep-disordered breathing in overweight and obese children and adolescents: prevalence, characteristics and the role of fat distribution. Arch Dis Child 2007; 92 : 205-8.

18. Costa DJ, Mitchell R. Adenotonsillectomy for obstructive sleep apnea in obese children: a meta-analysis. Otolaryngol Head Neck Surg 2009; 140: 455-60.

19. Burstein DH, Jackson A, Weedon J, Graw-Panzer KD, Fahmy S, Goldstein NA. Adenotonsillectomy for sleep-disordered breathing in a predominantly obese pediatric population. Int J Pediatr Otorhinolaryngol 2013; 77: 525-9.

20. Xu Z-F, Cheuk DKL, Lee S-L. Efficacy of tonsillectomy and adenoidectomy in Asian childhood obstructive sleep apnea/hypopnea syndrome: a case control study. Zhongguo Dang Dai Er Ke Za Zhi 2006; 8: 1-5.

21. Mitchell RB, Kelly J. Outcome of adenotonsillectomy for obstructive sleep apnea in obese and normal-weight children. Otolaryngol Head Neck Surg 2007; 137: 43-8.

22. Imanguli M, Ulualp SO. Risk factors for residual obstructive sleep apnea after adenotonsillectomy in children. Laryngoscope 2016; 126: 2624-9.

23. Com G, Carroll JL, Tang X, Melguizo MS, Bower C, JambHeKAR $S$. Characteristics and Surgical and Clinical Outcomes of Severely Obese Children with Obstructive Sleep Apnea. J Clin Sleep Med 2015; 11: 467-74.

24. Lee C-H, Hsu W-C, Chang W-H, Lin M-T, KANG K-T. Polysomnographic findings after adenotonsillectomy for obstructive sleep apnoea in obese and non-obese children: a systematic review and meta-analysis. Clin Otolaryngol 2016; 41: 498-510.

25. Lennon CJ, Wang RY, Wallace A, Chinnadurai S. Risk of failure of adenotonsillectomy for obstructive sleep apnea in obese pediatric patients. Int J Pediatr Otorhinolaryngol 2017; 92: 7-10.

26. Chang T-S, Chiang RP-Y. Total analysis of clinical factors for surgical success of adenotonsillectomy in pediatric OSAS. Eur Arch Otorhinolaryngol 2017; 274: 561-6.

27. Kheirandish-Gozal L, Gileles-Hillel A, AlonsoÁlvarez ML, Peris E, Bhattacharuee R, Terán-Santos J, ET AL. Effects of adenotonsillectomy on plasma inflammatory biomarkers in obese children with obstructive sleep apnea: A community-based study. Int J Obes 2015; 39: 1094-100.

28. Chu L, YaO H, Wang B. Impact of Adenotonsillectomy on High-Sensitivity C-Reactive Protein Levels in Obese Children with Obstructive Sleep Apnea. Otolaryngol Neck Surg 2012; 147: 538-43.

29. CHu L, LI Q. The evaluation of adenotonsillectomy on TNF- $\alpha$ and IL- 6 levels in obese children with obstructive sleep apnea. Int $J$ Pediatr Otorhinolaryngol 2013; 77: 690-4.

30. Gileles-Hillel A, Alonso-Álvarez ML, KheirandishGozal L, Peris E, Cordero-Guevara Ja, TeránSantos J, et al. Inflammatory Markers and Obstructive Sleep Apnea in Obese Children: 
The NANOS Study. Mediators Inflamm 2014; 605280: 1-9.

31. Gleich SJ, Olson MD, Sprung J, Weingarten tN, Schroeder DR, Warner D0, et al. Perioperative outcomes of severely obese children undergoing tonsillectomy. Pediatr Anesth 2012; 22: 1171-8.

32. Nafiu 00, Shanks A, Abdo S, Taylor E, Tremper TT. Association of high body mass index in children with early post-tonsillectomy pain. Int J Pediatr Otorhinolaryngol 2013; 77: 256-61.

33. Kshirsagar R, Mahboubi H, Moriyama D, AjosePopoola 0, Pham NS, Ahuja GS. Increased immediate postoperative hemorrhage in older and obese children after outpatient tonsillectomy. Int J Pediatr Otorhinolaryngol 2016; 84: 119-23.

34. Allareddy V, Martinez-Schlurmann N, Rampa S, Nalliah RP, Lidsky KB, Allareddy V, et al. Predictors of Complications of Tonsillectomy With or Without Adenoidectomy in Hospitalized Children and Adolescents in the United States, 2001-2010: A Population-Based Study. Clin Pediatr (Phila) 2016; 55: 593-602.

35. Lavin JM, ShaH RK. Postoperative complications in obese children undergoing adenotonsillectomy. Int J Pediatr Otorhinolaryngol 2015; 79: 1732-5.

36. Nafiu 00, Chimbira WT, Woolford SJ, Tremper KK, Reynolds PI, Green GE. Does High BMI Influence Hospital Charges in Children Undergoing Adenotonsillectomy? Obesity 2008; 16: 1667-71.

37. Katz ES, Moore RH, Rosen CL, Mitchell RB, Amin R, Arens R, et AL. Growth After Adenotonsillectomy for Obstructive Sleep Apnea: An RCT. Pediatrics 2014; 134: 282-9.

38. Van M, Khan I, Hussain SSM. Short-term weight gain after adenotonsillectomy in children with obstructive sleep apnoea: systematic review. $J$ Laryngol Otol 2016; 130: 214-8.

39. Levi J, Leoniak S, Schmidt R. Evaluating tonsillectomy as a risk factor for childhood obesity. Arch Otolaryngol Head Neck Surg 2012; 138: 897-901.

40. Topal K, Kara CO, Bozkurt Al, Saatci E. The risk of overweight and obesity in children after tonsillectomy: a cross-sectional study. Eur Arch Otorhinolaryngol 2013; 270: 689-94.

41. JUNAEB. Mapa nutricional 2017.

42. www.minsal.cl. MINSAL. Encuesta nacional de salud 2017.

43. Kukwa W, Kukwa A, Galazka A, Czarnecka AM, Krzeski A, Migacz E, et al. Long-term parental satisfaction with adenotonsillectomy: a population study. Sleep Breath2015; 19: 1425-9.

44. KIIER EK, Bock T, TIngsgaard PK. Tonsillotomy in children with sleep-disordered breathing is safe and results in high parent satisfaction. Dan Med J 2016; 63: A5228.

45. Afolabi OA, Alabi BS, Ologe Fe, Dunmade ad, SEgun-BusarI S. Parental satisfaction with postadenotonsillectomy in the developing world. Int J Pediatr Otorhinolaryngol 2009; 73: 1516-9.

46. Kovacevic L, Wolfe-Christensen C, Lu H, Lulguuraj M, Abdulhamid I, Thottam PJ, et al. Adenotonsillectomy improves quality of life in children with sleep-disordered breathing regardless of nocturnal enuresis outcome. $J$ Pediatr Urol 2015; 11: 269.e1-5.

47. Lee C-H, Kang K-T, Weng W-C, Lee P-L, Hsu W-C. Quality of life after adenotonsillectomy for children with sleep-disordered breathing: a linear mixed model analysis. Int $J$ Pediatr Otorhinolaryngol 2014; 78: 1374-80.

Dirección: Gerardo Oberreuter Espinoza 